\title{
Circuit
}

Musiques contemporaines

\section{L'écoute du Telharmonium 2.0 de Xavier Ménard et Camille St-Amand : les technologies électromécaniques au sein de l'espace d'exposition}

\section{Listening to Telharmonium 2.0 by Xavier Ménard and Camille St-Amand: Electromechanical Technologies in the Exhibition Space}

\author{
Karine P. Bouchard
}

Volume 30, numéro 1, 2020

URI : https://id.erudit.org/iderudit/1069084ar

DOI : https://doi.org/10.7202/1069084ar

Aller au sommaire du numéro

Éditeur(s)

Circuit, musiques contemporaines

ISSN

1183-1693 (imprimé)

1488-9692 (numérique)

Découvrir la revue

Citer cet article

Bouchard, K. P. (2020). L'écoute du Telharmonium 2.0 de Xavier Ménard et Camille St-Amand : les technologies électromécaniques au sein de l'espace d'exposition. Circuit, 30(1), 65-76. https://doi.org/10.7202/1069084ar
Résumé de l'article

Cet article interroge la manière dont l'installation d'art sonore Telharmonium 2.0 (2019) de Xavier Ménard et Camille St-Amand redéfinit notre rapport aux technologies électromécaniques par le biais de l'expérience d'écoute de l'oeuvre dans l'espace d'exposition. À partir d'une analyse qui fait interagir le discours des artistes au sujet de leur oeuvre, la restitution descriptive des matériaux constituants l'installation et l'expérience vécue de l'exposition, cet article propose que le Telharmonium 2.0 revisite doublement les interactions entre l'humain et la machine : d'une part, par les artistes qui exploitent le potentiel de la musicalité du bruit du moteur électromécanique ; d'autre part, par l'expérience d'écoute du visiteur de l'exposition qui réévalue auditivement cette musicalité. Se dégage alors une réflexion actualisée concernant l'esthétisation de cette mécanique par l'art sonore. 
CAHIER D’ANALYSE

\section{L'écoute du Telharmonium 2.0 de Xavier Ménard et Camille St-Amand: les technologies électromécaniques au sein de l'espace d'exposition}

Karine P. Bouchard

«Quelle est la nature des bruits que l'on écoute?» C'est la question que se pose d'emblée le visiteur de l'œuvre installative Telharmonium 2.o de Xavier Ménard et Camille St-Amand lorsqu'il entre dans la salle d'exposition pour y apercevoir trois sculptures sur socle (Figure 1). Si le visiteur est d'abord absorbé par le visuel esthétisant de l'installation, l'expérience contemplative, par l'œil, est rapidement contrariée par différentes textures sonores - rappelant des bruits industriels d'une technologie ancienne, de moteurs électriques - diffusées librement dans l'espace d'exposition'1.

Présentée dans le cadre du Festival International de Musique Actuelle de Victoriaville du 13 au 19 mai 2019, au milieu d'une salle adjacente à la bibliothèque Charles-Édouard-Mailhot, cette installation d'art sonore, une collaboration entre un compositeur et une designer industrielle, est le second volet d'une création en trois phases. La première partie, intitulée On/ Off $(1,0)$, a été exposée au Centre Clark du 10 mai au 16 juin $2018^{2}$ et la troisième demeure à ce jour en processus de réalisation. Selon les artistes, le fil conducteur qui guide la création de ce triptyque consiste à examiner, dans un contexte d'exposition d'art sonore, la manière dont les nouvelles technologies peuvent être utilisées au profit d'une mise en valeur de certaines technologies anciennes ${ }^{3}$. Par le biais du sonore, les artistes souhaitent, dans leur pratique, faire l'éloge d'éléments technologiques oubliés ou rarement considérés qui s'inscrivaient pourtant au cœur du fonctionnement des machines ayant
1. Cette description est issue de mon expérience personnelle de l'œuvre lors de ma visite de l'exposition, le 17 mai 2019.

2. Pour des informations supplémentaires sur cette œuvre, consulter le site web du Centre Clark: centreclark.com/fr/ exposition/on-off-10/ (consulté le 13 novembre 2019). On/Off (1,0) a également été présentée dans le cadre d'Art souterrain en mars 2017 et au Centre d'exposition de l'Université de Montréal en mai 2018.

3. Sauf indication contraire, les propos attribués à Ménard et Saint-Amand au cours de cet article sont tirés d'un entretien mené le 13 juin 2019. 
servi aux développements de l'industrialisation des débuts du siècle dernier, dont le moteur électrique, cet appareil transformant de l'énergie afin qu'elle devienne mécanique.

À partir de ce postulat, j'interrogerai la manière dont l'œuvre installative de Ménard et St-Amand favorise une expérience actuelle, et surtout musicale, des technologies anciennes ainsi que du moteur électrique, plus précisément. Je considère que la mise en espace de l'installation et le contexte d'exposition, en stimulant notre écoute, conduit précisément à cette actualisation. En faisant interagir la proposition théorique des artistes, la reconstitution descriptive des matériaux constituant l'installation et ma propre expérience de l'exposition, j'examinerai l'œuvre sous trois aspects : 1) j’analyserai de façon détaillée le fonctionnement même de l'installation dans son lieu d'exposition afin de comprendre la démarche singulière sous-jacente, qui consiste en

FIGURE 1 Xavier Ménard et Camille St-Amand, Telharmonium 2.0 (2019). Crédit: Camille St-Amand.

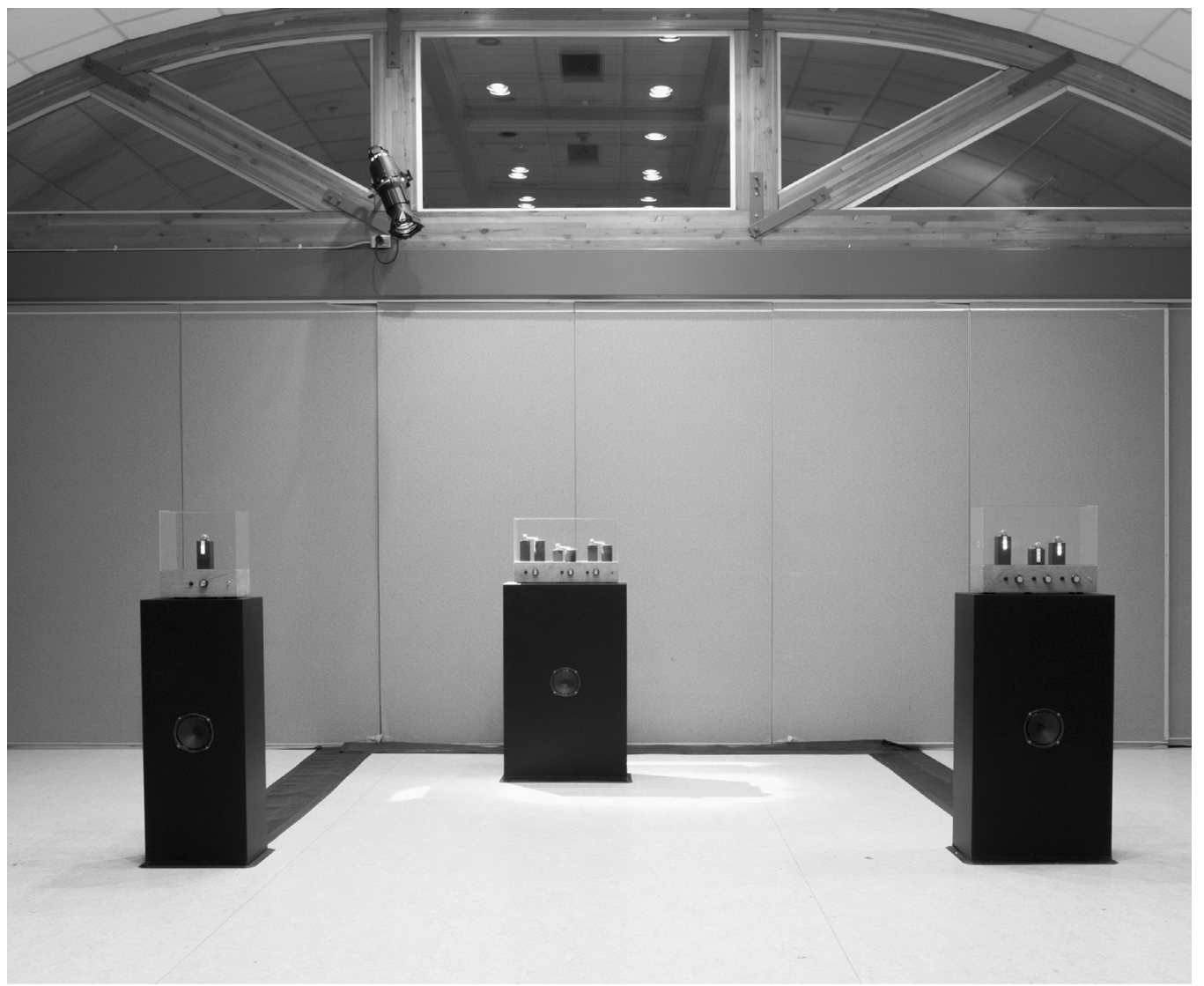


une composition mettant en interaction le visuel, la matérialité, le sonore, l'espace et les visiteurs ${ }^{4}$; 2) il s'agira d'évaluer cette composition en fonction d'une musicalité du bruit; 3) je chercherai à comprendre comment l'œuvre favorise, par une mise en espace spécifique, des interactions visuelles et sonores avec les visiteurs. L'étude soulignera principalement les expériences d'écoute des bruits des technologies électromécaniques dans un contexte qui leur est généralement étranger, soit l'environnement de l'exposition artistique. En définitive, cette analyse m’amènera à réfléchir au potentiel artistique et musical des technologies anciennes et électromécaniques en général.

\section{L'installation : de la matérialité à la composition musicalisée}

\section{a) Mise en espace}

À sa première présentation, laquelle sera retenue pour les besoins de ce texte, l'œuvre a été exposée dans le cadre du programme d'installations sonores présentées dans l'espace public par le FIMAV, organisé par le commissaire Érick d'Orion. Lors de l'édition 2019, les œuvres sélectionnées spécifiquement pour l'occasion ont été créées autant par des musiciens électroacoustiques que par des artistes visuels ${ }^{5}$.

En entrant dans la salle d'exposition destinée à l'installation de Xavier Ménard et de Camille St-Amand, le visiteur aperçoit, adjacent à l'une des quatre cimaises épurées, le Telharmonium 2.o (Figure 1) ${ }^{6}$. L’œuvre sculpturale, qui se veut «visuellement agréable», pour reprendre les termes des artistes, comporte trois modules placés à égale distance l'un de l'autre de manière à s'imposer frontalement au public. La structure du milieu, légèrement en retrait et plus près de la cimaise, crée un effet de point de fuite. Chaque module repose sur un socle rectangulaire noir avec une enceinte intégrée, imitant l'apparence d'un vieil amplificateur. Sur le socle se trouvent des moteurs électriques miniatures en mouvement; ceux-ci produisent des sons de manière autonome, sous une cloche en verre rectangulaire? ${ }^{7}$ Le module de gauche ne contient qu'un seul moteur, tandis que celui du centre et celui de droite en présentent trois. Chaque moteur est placé sur un piédestal, dont la hauteur varie selon l'appareil, garni d'une lumière qui sallume par intermittence, en interaction avec les éléments cinétiques des mécanismes. Sous ce système, un cadre en bois rend accessible au visiteur des boutons servant à activer les moteurs. Enfin, deux haut-parleurs surélevés, accrochés à la cimaise opposée de la pièce, complètent l'installation. Le visuel de cette œuvre s'inscrit dans la continuité des recherches entamées lors de la première phase du triptyque, matérialisée par l'installation On/Off (1,o), où l'idée du socle et de boutons activés par les visiteurs est récurrente dans la conception et la mise en espace.
4. Ici, la notion de composition ne renvoie pas uniquement à la musique, mais bien à l'ensemble de l'œuvre.

5. Parmi les artistes de l'exposition se trouvent Émilie Payeur, L'orchestre d'hommes-orchestres, le collectif Scenocosme, Ludovic Boney et Georges Azzaria. Pour des précisions concernant les œuvres, voir: www. fimav.qc.ca/fr/edition/installationssonores-dans-I-espace-public (consulté le 26 novembre 2019).

6. Les artistes souhaitent remercier Francis Lecavalier pour la programmation Arduino et l'atelier du Centre Clark pour la construction des modules.

7. La cloche est un dispositif couramment utilisé dans les expositions d'arts visuels de musées. Bien que la structure ici soit rectangulaire, le terme "cloche" est d'usage courant. 
8. Weidenaar (1995, p. xv) recense trois variantes du telharmonium: un prototype réalisé en 1900 ainsi que deux versions commerciales en 1906 et 1911.

9. Pour une analyse détaillée du fonctionnement du Telharmonium, consulter Holmes, 2016.

10. Centre Clark, 2018.

\section{b) Fonctionnement}

Comme son titre l'indique, le projet du duo s'inspire de l'instrument éponyme - le telharmonium, instrument de musique électromécanique développé par Thaddeus Cahill à partir de 1893 aux États-Unis - et explore les possibilités d'autonomisation de la machine pour l'exécution musicale ${ }^{8}$. Dans la lignée de l'orgue, le telharmonium produit des sons complexes suivant le procédé de synthèse additive9. Ménard et St-Amand ont retenu de ce dernier l'idée d'activation de moteurs par des impulsions électriques pour produire le son et générer des fréquences ${ }^{10}$.

Dans l'espace d'exposition, le premier module de l'installation (à gauche, dans la Figure 1) contient un moteur générateur de sons plus imposant que les deux autres: il incarne la basse de l'ensemble. Le second module (à droite, dans la Figure 1), constitué de trois moteurs générateurs de sons, est conçu pour la composition de la mélodie. Trois moteurs pas à pas forment le dernier module (au centre de l'espace, dans la Figure 1), qui joue le rôle de la section rythmique. Ce troisième module, en retrait, au centre de l'installation, fonctionne différemment des deux autres: c'est un activateur constitué de mécanismes qui déclenchent des pistons sur des membranes, lesquelles font

FIGURE 2 Xavier Ménard et Camille St-Amand, Telharmonium 2.0 (2019), détail du module du centre. Crédit: Camille St-Amand.

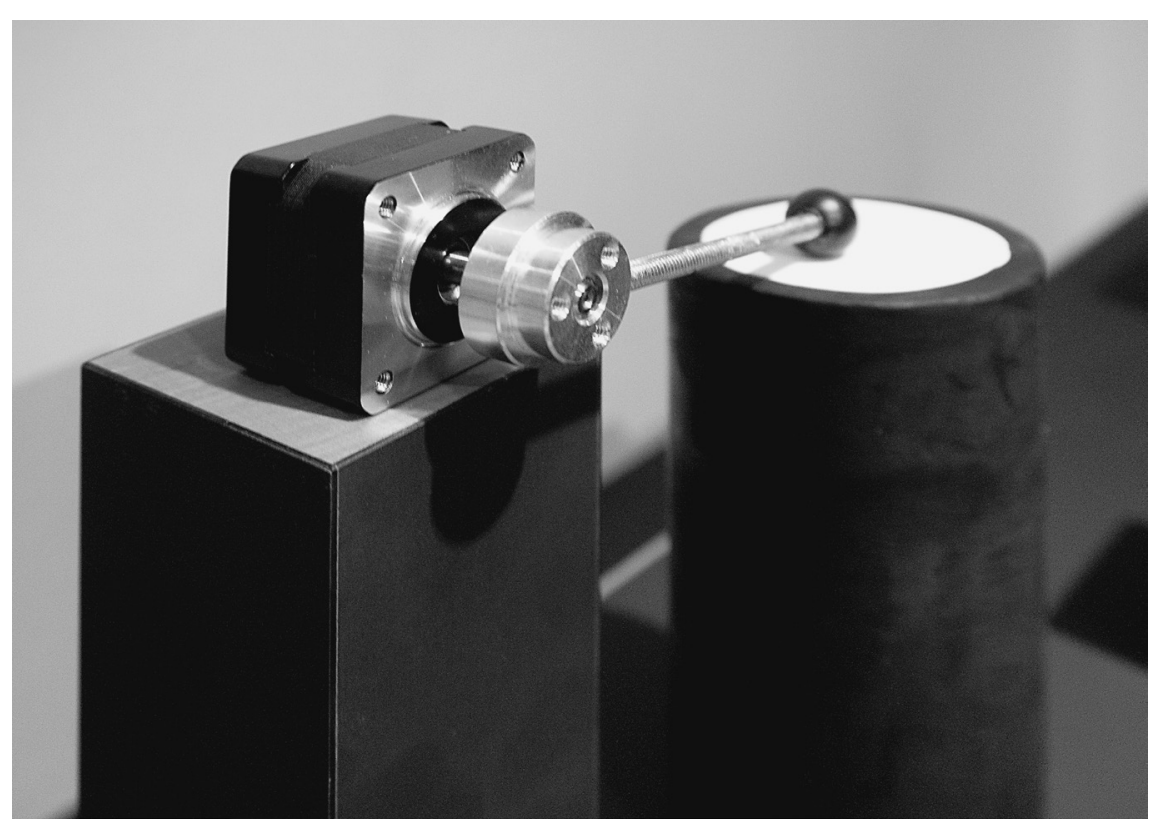


office de tambour (Figure 2). Le rythme percussif est ensuite capté par des microphones de contact ${ }^{11}$.

Concernant l'émission du son, ce «rôle est laissé aux machines», selon Ménard et St-Amand. Tous les sons entendus, d'une durée aléatoire, sont produits physiquement par les moteurs. Ils s'apparentent à des bruits mécaniques et électriques, soit des bruits de frottement de pièces de métal, entre autres.

Linstallation est composée de quatre consoles de mixage, dont une principale, chacune associée à un module et à des microcontrôleurs Arduino pour créer une composition musicalisée (Figure 3). Par une connexion Wi-Fi, le contrôleur Arduino principal achemine les séquences composées aux trois microcontrôleurs imbriqués dans les modules. Ce système règle la vitesse d'accélération des moteurs et, par conséquent, la hauteur et le volume des sons produits: plus le mécanisme du moteur s'active, plus le son devient aigu et fort.
11. Concernant les dimensions des socles, leur largeur varie ainsi : le socle de la basse mesure $35 \mathrm{~cm}$; celui de la mélodie, $50 \mathrm{~cm}$, et des percussions, $60 \mathrm{~cm}$. Les socles font tous $25 \mathrm{~cm}$ de profondeur et $110 \mathrm{~cm}$ de hauteur. Chaque module dispose également d'une largeur spécifique: le module de la basse mesure $30 \mathrm{~cm}$ et celui de la mélodie, $45 \mathrm{~cm}$; les proportions du module de percussions sont de $55 \mathrm{~cm}$. Tous ont les mêmes hauteur $(35 \mathrm{~cm})$ et profondeur $(20 \mathrm{~cm})$.

FIGURE 3 Xavier Ménard et Camille St-Amand, Telharmonium 2.0 (2019), schéma du dispositif. Schéma : Xavier Ménard.

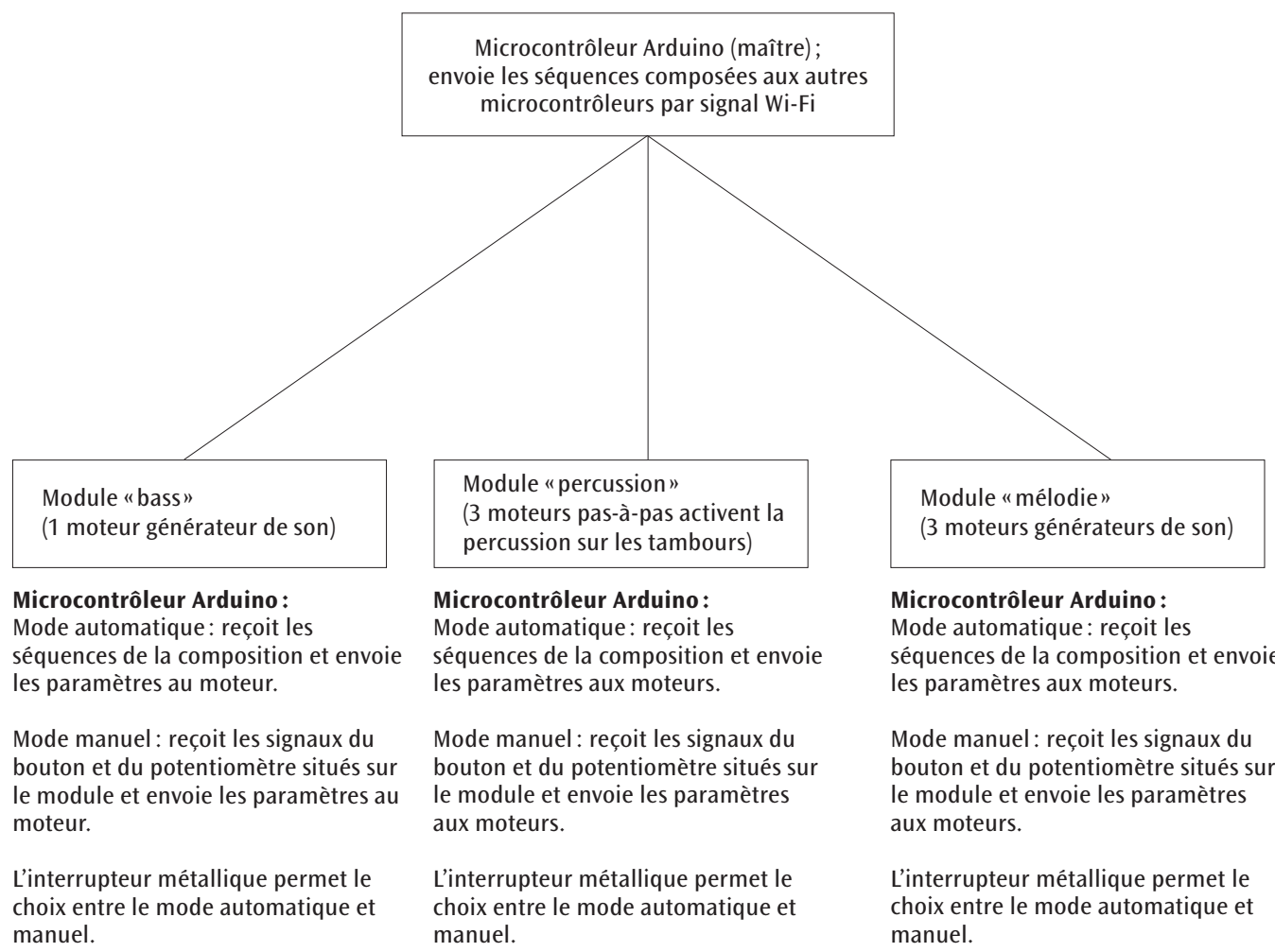


FIGURE 4 Xavier Ménard et Camille St-Amand, Telharmonium 2.0 (2019), module de percussion. Crédit: Camille St-Amand.

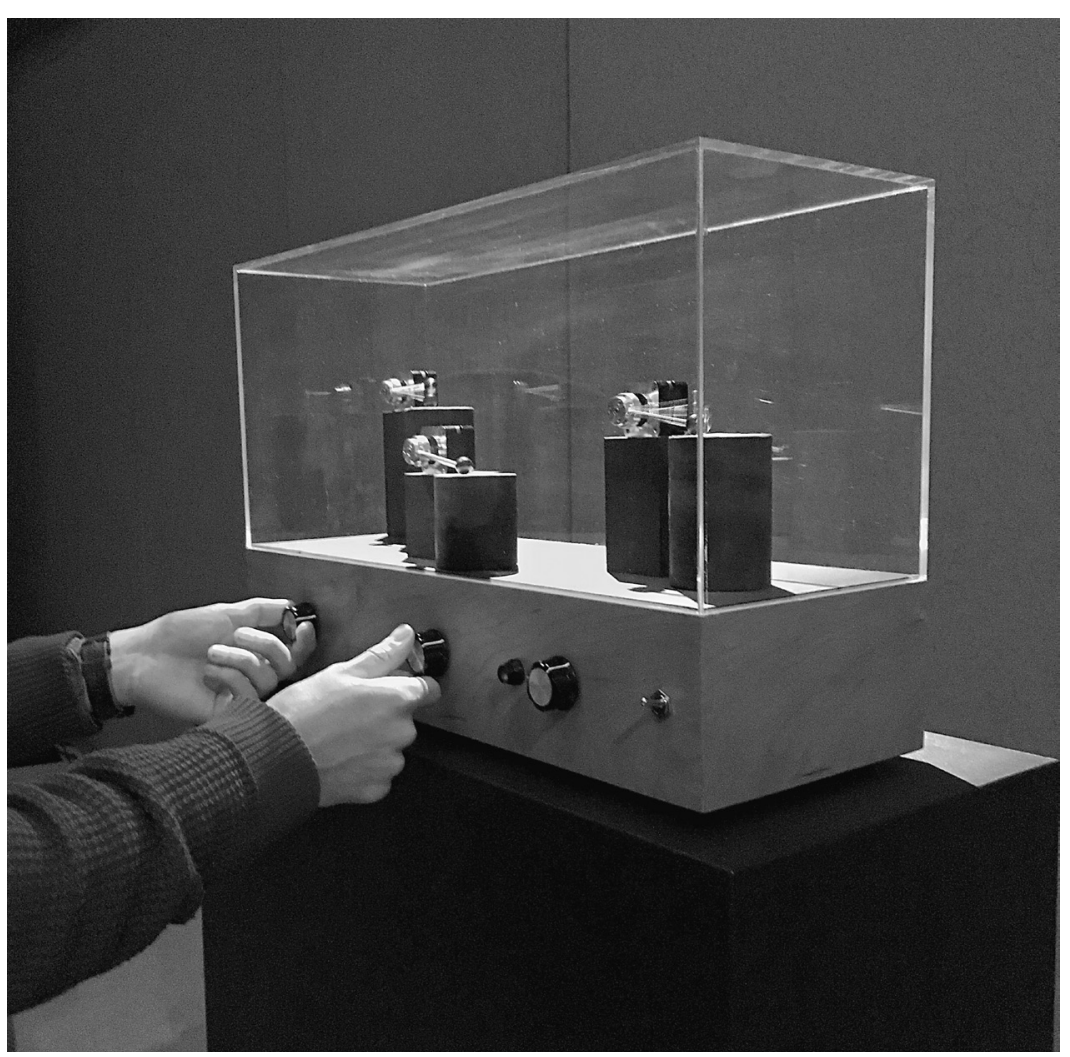

Concrètement, lorsqu'ils entrent dans la salle, les visiteurs écoutent d'abord une pièce d'environ 45 secondes qui joue en boucle, puisque les microcontrôleurs Arduino des modules sont programmés en mode automatique: ils reçoivent les séquences de la composition issue du contrôleur principal et transmettent les paramètres au moteur. Les sons sont ainsi générés par la machine et se mixent entre eux de façon aléatoire. Une rythmique tantôt synchronisée, tantôt en déphasage se développe en temps réel. Les visiteurs peuvent ainsi avoir l'impression d'écouter une composition pour un ensemble à trois parties dans la mesure où les modules fonctionnent de concert, formant ainsi une sorte d'orchestre réduit.

À tout moment, ce réglage par défaut et ce mixage peuvent être altérés par les visiteurs en manipulant les boutons à leur disposition : ils sont amenés à tourner vers la droite ou vers la gauche un ou plusieurs boutons correspondant 
au moteur de leur choix dans le but de modifier les fréquences. Cette activation manuelle, qui s'effectue à l'aide d'un potentiomètre intégré à la structure, vient ébranler l'équilibre musical préprogrammé de la composition initiale. Pour choisir entre les modes automatique et manuel, un interrupteur métallique, situé à côté des boutons sur chaque structure, est à la disposition des visiteurs (Figure 4). Enfin, les différentes compositions sont diffusées à la fois par les haut-parleurs des modules et tous les sons des moteurs, amplifiés, sont simultanément entendus par le biais des haut-parleurs situés du côté opposé de la pièce. L'installation offre ainsi une expérience d'écoute essentiellement dans le lieu physique et en temps réel, où les sons diffusés qui se mélangent à ceux produits par les visiteurs (bruits de pas, chuchotements, etc.) résonnent et se réverbèrent dans l'espace.

\section{Le nouveau au service de l'ancien}

\section{a) La réactualisation des technologies électromécaniques}

Comme mentionné plus haut, les artistes s'inspirent du fonctionnement du telharmonium du XIX siècle pour réfléchir aux possibilités de générer des fréquences grâce à un système de moteurs électriques. Pour ce faire, ils organisent ces moteurs en réseaux, tout en supprimant le clavier de l'instrument initial ainsi que le système de roue phonique au profit d'un microcontrôleur Arduino. De cette manière, ils revisitent le potentiel mécanique de l'instrument à la lumière des avancées actuelles du numérique:

En tant que compositeurs, nous sommes particulièrement intéressés à faire revivre un instrument électromécanique centenaire dans une époque de musique numérique dématérialisée. Nous voulons modifier ses caractéristiques, le moderniser grâce à des technologies d'aujourd'hui dans une perspective transhistorique, c'est-à-dire faire cohabiter des éléments d'origine avec d'autres plus contemporains ${ }^{12}$.

12. Ménard, 2019.

Ce changement radical que les artistes apportent à l'instrument du tournant du $\mathrm{Xx}^{\mathrm{e}}$ siècle se traduit par une installation qui doit être envisagée comme «un hybride des technologies passées et actuelles ${ }^{13}$ ».

Au-delà d'une référence à l'instrument de Cahill, cette réflexion historique sur les technologies électromécaniques est pensée de manière élargie par les artistes, qui rappellent que le moteur est aux fondements de toute machine, dès l'arrivée de l'industrialisation. Cette idée était déjà présente lors de la réalisation de On/Off $(1,0)$ : «Depuis l'ère industrielle, la logique des chaînes de montage est basée sur le On/Off, logique qui permettait de faire démarrer différents procédés. À l'ère numérique, celle-ci est connue sous la forme binaire $(1,0)^{14}$.» Si elles ont été développées en masse dès les débuts de l'ère 
15. Ibid.

16. Ménard, 2019. industrielle, les machines sont devenues aujourd'hui omniprésentes et ont des usages et des sons variés: machine-outil dans les chaînes de montage, robots, imprimante $3 \mathrm{D}$, drone, etc. Les artistes postulent à cet effet que nous sommes à « une ère de globalisation industrielle où la main-d'œuvre est remplacée par des technologies robotisées jusque dans les détails les plus banals de la vie (les points de service dans les restaurants rapides, les caisses en libre-service à l'épicerie, le guichet automatique à la station de métro $)^{15}$ ». L'impact de la machine sur les habitudes de l'humain est récurrent selon le duo. Il est ainsi important, pour eux, de réfléchir au fonctionnement des moteurs mécaniques lorsqu'ils sont présentés hors de leur contexte d'origine, lié à la production, soit lorsqu'ils sont récupérés au sein d'un processus de création artistique qui les esthétise. C'est pourquoi le Telharmonium 2.o propose aux visiteurs une expérience visuelle, sonore et tactile inédite du moteur en lui-même, dans une visée performative.

Par la réalisation de l'installation, le duo a transformé les moteurs en un (nouvel) instrument de musique qui «réunit la technologie industrielle d’une autre époque, l'automatisation de procédés par langage de programmation et la possibilité du contrôle complet de la technologie par l'interaction humaine ${ }^{16}$ ». Si les rapports entre la mécanique et le visiteur ont constitué les premières préoccupations des artistes, la prise en compte des bruits produits par le moteur et leur spatialisation sonore dans le lieu d'exposition est ensuite devenue cruciale à la réalisation matérielle et à la réussite de l'expérience de l'œuvre.

\section{b) L'éloge du (bruit du) moteur}

Ménard et St-Amand souhaitent mettre en valeur l'aspect physique et sonore des moteurs lorsqu'ils ne sont plus tributaires de l'activation d'une machine. Le duo fait remarquer que ces technologies électromécaniques, bien qu'elles aient été omniprésentes dans la société, ont rarement fait l'objet de considérations particulières: «on n'y porte jamais vraiment attention». Elles sont le plus souvent subordonnées ou camouflées de manière à servir, par l'électricité notamment, différents appareils autres qu'eux-mêmes. Au sein du Telharmonium 2.o, les artistes soulignent que ces moteurs sont montés sur de petits podiums pour présenter la nature précise de leur mécanique: «nous souhaitons garder le naturel du moteur, sans le cacher, ni l'habiller». En s'émancipant de tout accessoire et ornement, les moteurs apparaissent dans leur intégrité, dénudés de tout dispositif.

Toujours selon le duo, la présentation de ces moteurs électromécaniques sous une cloche d'exposition, tels des artefacts historiques, sert à "protéger cette ancienne technologie ${ }^{17} »$. Il importe de rappeler que les musées d'art 
visuel ont souvent fait usage du dispositif de cloche de verre pour préserver l'artefact, devenu œuvre d'art, hors du temps et de l'espace, tout en abolissant sa fonction première ${ }^{18}$. Dans le cas des modules du Telharmonium 2.0, cette logique permet de croire que la cloche élève au rang d'œuvre d'art les moteurs qui sont alors contemplés par les visiteurs.

Cette volonté d'exhiber la mécanique ébranle les pratiques actuelles où les éléments en monstration sont plutôt issus des nouvelles technologies et du numérique. Contrairement à cette tendance, les technologies actuelles du Telharmonium 2.o sont camouflées pour laisser place à la présentation visuelle de la composante mécanique, le moteur.

Toutefois, le visuel est rapidement mis au profit du sonore de manière à encourager l'éloge des bruits, car ce qui est véritablement exposé utilise le visible pour ensuite conduire à une esthétisation de ces sons. Grâce à des transducteurs de petits formats qui captent les occurrences sonores et permettent leur diffusion ainsi que leur spatialisation dans la salle, le Telharmonium 2.0 transgresse toute contemplation visuelle passive des moteurs en diffusant leurs bruits hors de la limite physique de la cloche de verre.

\section{Expérience(s) de l'exposition : interactions spatiales, sonores et sociales}

\section{a) La musicalité du bruit}

Les bruits de l'installation sont changeants, parfois inattendus, persistants et immersifs. Ils forment entre eux des séquences rythmées, indépendantes, chacune ayant son motif et, par conséquent, sa spécificité. Plus précisément, la juxtaposition de modifications rapides de la hauteur des sons, passant d'un son grave à un son aigu et strident, activées entre autres par les accélérations et décélérations des moteurs, favorise des compositions musicalisées singulières. De ces enchaînements ainsi émerge une musicalité. Comme l'expliquent les théoriciens et praticiens du cinéma Serge Cardinal, Martin Allard et Louis Comtois, la musicalité se construit dans la relation entre le fragment et le tout, entre la plasticité des sons et leur organisation d'ensemble:

[L]es sons (pris cette fois dans leur forme globale) se lient les uns aux autres, s'agglutinent, se superposent, créent des lignes, des paliers, des épaisseurs, des séquences. La musicalité vient donc à la fois des qualités plastiques des sons et de la complexité de leur organisation ${ }^{19}$.

La musicalité du bruit est un produit de l'engagement actif des spectateurs, qui prennent part à l'organisation des sons et cherchent, avec une écoute active, les rapports de sens entre le tout et les parties. Ainsi, c'est la musicalité
18. Par exemple, ce fut le cas de l'exposition Art by Telephone Recalled (La Panacée - Centre de culture contemporaine de Montpellier, France), organisée par les commissaires Sébastien Pluot et Fabien Vallos en 2013, où un tel dispositif servait à montrer un disque vinyle qui était le catalogue de l'exposition Art by Telephone (Museum of Contemporary Art Chicago, 1969), à l'origine de l'événement de 2013. Sous cette cloche de verre, le vinyle perdait sa fonction de diffusion du son, n'étant ni activé par un tourne-disque ni manipulé par les visiteurs. La cloche le réduisait à l'état d'objet silencieux, offert pour la contemplation. Voir Bouchard (2015).

19. Cardinal, Allard et Comtois, 2002, p. 172. 
20. Centre Clark, 2018.

21. Lacey, 2013, p. 72, ma traduction.

22. Ibid du bruit, lequel circule en réverbérant et en résonant dans l'espace d'exposition, qui rend efficace et cohérent l'ensemble du Telharmonium 2.o.

\section{b) L'organisation sociale par l'écoute}

Par leur visuel «invitant» qui, selon les artistes, rappelle l'esthétique des technologies des années 1960 et 1970, On/Off $(1,0)$ et Telharmonium 2.0 «intriguent» les visiteurs qui expérimentent ces œuvres en se dirigeant vers les structures installatives pour les saisir de près: «nous sommes obligés de nous [en] approcher », confirme le duo. Si le visuel renvoie à une facilité d'entrer en contact physiquement avec l'œuvre, il suggère également, à un second niveau, le fait de rendre «accessible à tous la performance de musique électroacoustique », tel qu'il est mentionné dans la description de On/Off $(1,0)$ par le Centre Clark ${ }^{20}$. Dans le cas du Telharmonium 2.o, les manipulations offrent au public une expérience d'autant plus près d'une réelle performance de musique électroacoustique. Par certaines manipulations, les visiteurs peuvent, à leur guise, prendre le contrôle des sons, altérer la composition initiale et intervenir sur l'organisation des fréquences de la basse, la hauteur de la mélodie et les rythmes des moteurs et des tambours. En découle de cet environnement acoustique une expérience où l'oreille du visiteur devient la clé de voûte de cette musicalité et guide l'orientation de la composition.

Cette musicalité amène à réfléchir aux nouvelles organisations sociales possibles dans lesquelles les bruits s'imposent au corps et à la pensée, comme le propose la théoricienne et historienne des médias Kate Lacey: «L'incapacité de fermer nos oreilles aux sons qui nous entourent semble rendre l'audition plus sensible que la vision, plus vulnérable aux contraintes du monde extérieur. [...] Le bruit est immatériel, pourtant, il peut envahir le corps et coloniser l'esprit ${ }^{21}$. Cette référence à la « colonisation » suggère que le son incarne un pouvoir d'organisation des relations sociales, selon les perceptions acquises culturellement, tel que le souligne Lacey: «La sensibilité au son varie avec le temps, selon les cultures et entre les classes sociales, et ainsi la manière dont le paysage sonore est perçu et géré en dit long sur la structuration des relations sociales ${ }^{22}$.»

Plus concrètement, dans l'espace d'exposition, cette organisation des relations sociales se construit grâce à l'écoute des bruits et se traduit par les interactions entre les spectateurs et les modules, mais aussi entre les différents individus. Ces interactions rappellent l'expérience de faire de la musique en groupe, puisque les visiteurs «jouent» simultanément et peuvent s'ajuster à l'oreille aux transformations musicales improvisées de chacun d'eux. Le visiteur incarne à la fois l'interprète et le compositeur en écoutant les sons qu'il 
crée lui-même et en répondant à ces occurrences sonores par l'improvisation. Surtout, il est amené à réagir aux sons qui proviennent des autres modules et qui sont constamment modifiés par les différents visiteurs.

Lexposition de la version 2.o participe donc à la réinvention du telharmonium de Cahill de sorte qu'il puisse être manipulé par le non-spécialiste, comme le spécifie le duo d'artistes: «L'absence de complexité d'utilisation de la machine permet une ouverture intergénérationnelle, c'est-à-dire qu'elle peut être manipulée par des gens de tous âges, de tous horizons, dans une optique ludique, inclusive et créative ${ }^{23}$.» L'œuvre favorise une initiation 23. Ménard, 2019. aux principes de la musique, dans une visée démocratique et accessible au plus grand nombre, par une appropriation directe des différents sons où les schèmes de la composition électroacoustique, qui suppose une démarche en studio ou à l'ordinateur, sont ici remplacés par l'improvisation et la manipulation facilitées et en direct d'un instrument électronique.

En somme, en découvrant le potentiel sonore de l'installation, les visiteurs tiennent le rôle de musicien. Leur corps s'organise dans l'espace par la volonté de participer, par l'écoute, à la composition. Une performance peut avoir lieu où l'«instrument» nécessite alors une pluralité d'interactions entre les artistes et le contrôleur électronique, entre le contrôleur et le moteur électrique, entre le public et le moteur, entre les créateurs et les visiteurs. Tous ces acteurs participent, ensemble, à la finalité de la composition musicalisée de l'installation d'art sonore.

\section{Conclusion}

C'est ainsi grâce au potentiel d'organisation spatiale que peut se développer une composition - musicalisée - à partir des différentes composantes installatives. Une musicalité des bruits de la technologie électromécanique se construit alors par l'écoute des visiteurs, activée par les éléments visuels et tactiles, dans une condition immersive et interactive.

L'œuvre revisite doublement les interactions entre l'humain et la technologie électromécanique: d'une part, par les artistes qui réactualisent le moteur, en réévaluant la connotation associée à son image; d'autre part, par le visiteur qui regarde, écoute et manipule la structure de manière à en dégager une réflexion actuelle sur son propre rôle vis-à-vis des technologies anciennes. La (ré)organisation des bruits pour former des compositions musicalisées affranchit cette œuvre d'art sonore d'une association trop mimétique ou historique aux sons de moteurs. Dans ce contexte artistique, Xavier Ménard et Camille St-Amand semblent offrir une expérience qui établit un équilibre entre le rythme des technologies électromécaniques, celui de la musique et 
celui de l'humain, adapté à la structure, aux normes, aux exigences et aux codes de l'exposition visuelle et sonore. Le moteur évolue alors au rythme de l'exposition, où l'installation autorise davantage une prise de contrôle de ses bruits et de sa musicalité par le visiteur.

L'installation de Ménard et St-Amand permet d'offrir une manière d'écouter les sons qui déconstruit les différentes relations inhérentes à tout système préétabli du bruit de la technologie électromécanique qui serait organisé par les sociétés et l'histoire. Les relations qui se (re)créent alors entre ces technologies, l'écoute de ses bruits et les visiteurs dans l'espace d'exposition apparaissent davantage égalitaires, où le pouvoir n’est pas uniquement accordé à une autorité (le commissaire ou les artistes), mais est distribué plus équitablement au profit des visiteurs, favorisant des postures d'échanges et un sentiment de collectivité chez les individus.

\section{B IB LI O G RAPHIE}

Bijsterveld, Karin (2012), «Listening to Machines: Industrial Noise, Hearing Loss and the Cultural Meaning of Sound », in Jonathan Sterne (dir.), The Sound Studies Reader, Londres/ New York, Routledge, p. 152-167.

Bouchard, Karine (2015), «Sound Recordings in the Art Gallery», Association for Recorded Sound Collections Journal, $\mathrm{n}^{\circ}$ 46, p. 77-85.

Cardinal, Serge, Allard, Martin et Comtois, Louis (2002), «La musicalité d'une bande sonore: à propos de L'Invention d'un paysage», in Réal Larochelle (dir.), Écouter le cinéma, Montréal, 400 coups, p. 158-174.

Holmes, Thomas (2016), Electronic and Experimental Music: Technology, Music, and Culture, New York/Londres, Routledge.

Lacey, Kate (2013), Listening Publics: The Politics and Experience of Listening in the Media Age, Cambridge, Polity.

WeidenaAr, Reynold (1995 [1989]), Magic Music From the Telharmonium, Metuchen, Scarecrow Press.

\section{ADRESSES URL}

Centre Clark (2018), «Xavier Ménard et Camille St-Amand», https://centreclark.com/fr/exposition/on-off-10/ (consulté le 13 novembre 2019).

MÉNARD, Xavier (2019), «Installations sonores », xavierm.ca/installations-sonores/ (consulté le 31 juillet 2019). 